\title{
REVIEW
}

\section{Definitions of dementia and predementia states in Alzheimer's disease and vascular cognitive impairment: consensus from the Canadian conference on diagnosis of dementia}

\author{
Howard Chertkow ${ }^{* 1,2}$, Howard H Feldman ${ }^{3}$, Claudia Jacova ${ }^{4}$ and Fadi Massoud ${ }^{5}$
}

\begin{abstract}
There have been several newly proposed sets of diagnostic criteria for Alzheimer's disease/mild cognitive impairment, advanced by the National Institute of Aging/Alzheimer's Association working groups in 2011 and by the International Working Group in 2007 and 2010. These sets each aim to provide broader disease stage coverage with incorporation of disease biomarkers into the diagnostic process. They have focused particular attention on the earlier identification of disease with focus on the preclinical and predementia stages. This paper reviews these diagnostic criteria and provides 2012 consensus recommendations from the Canadian Consensus Conference on the Diagnosis and Treatment of Dementia on their applications in both clinical and research settings.
\end{abstract}

\section{Introduction}

This report was prepared for the Canadian Consensus Conference on the Diagnosis and Treatment of Dementia (CCCD) held in May 2012 in Montreal, Canada. The purpose was to evaluate new definitions of dementia and predementia states in Alzheimer's disease (AD) and vascular cognitive impairment (VCI), and to provide consensus recommendations for their potential application in clinical and research settings of care that were discussed and voted on at the CCCD.

New diagnostic criteria for AD have been advanced recently by the National Institute of Aging/Alzheimer's Association (NIA-AA) working groups in 2011 and by

*Correspondence: howard.chertkow@mcgill.ca

²Bloomfield Centre for Research in Aging, Lady Davis Institute, 3755 Cote St Catherine Road, Montreal, Canada H3T 1E2

Full list of author information is available at the end of the article the International Working Group (IWG) led by Bruno Dubois in 2007 and 2010. Three NIA-AA workgroups reported new guidelines for diagnosis of dementia and $\mathrm{AD}$ [1], mild cognitive impairment (MCI) [2], and new definitions for the preclinical stages of AD [3]. The IWG proposed new diagnostic criteria and a new lexicon for AD including its preclinical and prodromal stages $[4,5]$. The IWG criteria were clearly stated to be research criteria. The NIA-AA committees concentrated largely on research but also defined new clinical guidelines for dementia states and MCI with consideration of their utility in the clinic. Both working groups aimed to integrate in vivo Alzheimer's pathological biomarkers into the diagnostic process

The potential for earlier $\mathrm{AD}$ diagnosis has been supported by the advent of promising in vivo cerebrospinal fluid (CSF) measures of amyloid-beta (A $\beta) 1$ to 40, $A \beta 1$ to 42 , total tau and phospho-tau, and neuroimaging biomarkers - structural magnetic resonance imaging (MRI), amyloid positron emission tomography (PET), fludeoxyglucose (FDG) PET - that reflect the presence of the $\mathrm{AD}$ pathophysiological process (AD-P). While these biomarkers enable the identification of AD-P in vivo, there is still much to learn about their significance in asymptomatic individuals and the associated increased risk of progression to $\mathrm{AD}$ dementia. $\mathrm{AD}-\mathrm{P}$ biomarkers have not yet reached regulatory approval in Canada and nor are they funded for use in clinical settings, yet they form an important opportunity to improve the accuracy of diagnosis in research studies across the disease stages.

Several important points should be noted. First, both the NIA-AA working group and the IWG criteria focus primarily on $\mathrm{AD}$, with consideration of its preclinical, predementia and dementia stages. The issues of differential diagnosis, diagnostic criteria for other non-AD dementias, and mixed dementias are beyond the scope of the criteria and of this review. Second, there is a deliberate effort to distinguish between the clinical syndromes pertaining to $\mathrm{AD}$, and the biomarker evidence of 
its pathologic process (AD-P). Other articles in this CCCD supplement will further address the developmental issues pertaining to AD-P biomarkers, while this paper focuses more centrally on clinical syndromes pertaining to AD. Third, within AD-P biomarkers, pre-eminence is given to amyloid pathology, given its early presence as a molecular neuropathological disease marker and the associated hypothesis that therapeutic strategies to enhance its clearance could be preventive if administered early enough in the disease process [6,7]. One must note that these models are evolving as conceptual frameworks and are still largely hypothetical at the present time.

This paper also includes a section on VCI. The American Heart Association and the American Stroke Association (AHA/ASA) recently formed a working group that has issued a consensus position paper entitled 'Vascular Contributions to Cognitive Impairment and Dementia' in 2011 [8]. This new proposed diagnostic framework was considered sufficiently comprehensive to warrant consideration for the Fourth CCCD.

\section{The syndrome of dementia}

Dementia is typically defined as a clinical syndrome of cognitive decline that is sufficiently severe to interfere with social or occupational functioning. It remains an anchor point of reference within the revised AD diagnostic criteria proposals. Routine clinical practice shows that the cognitive and functional changes of dementia are typically accompanied by changes in behavior and in personality, but these have not become core criteria as they have been considered heretofore to lack sufficient diagnostic specificity.

Dementia has been and remains a clinical diagnosis, in which laboratory or imaging tests as yet provide only supportive diagnostic evidence. Diagnosis includes a careful history. In specialist practice, this is often done using a semi-structured interview with an informant (for example, the Clinical Dementia Rating scale [9]) although a wide variety of assessment approaches exist. Diagnosis also involves a detailed medical and neurological examination, and a formal mental status examination including bedside cognitive testing. Brief assessment tools are often used (for example, the Montreal Cognitive Assessment [10]) whereas in some referral centers extensive neuropsychological testing is used as an ancillary diagnostic aid. Structured or informal functional assessment is used to establish the presence and severity of functional disability.

Dementia diagnoses are most commonly based on the Diagnostic and Statistical Manual of Mental Disorders, 4th edition, text revised criteria [11-13], which require impairment of memory and at least one of the following domains: language, praxis, gnosis, or executive functioning. These impairments need to be sufficiently severe to impair social or professional life, and must not occur as a consequence of a delirium, or be caused by another medical, neurological or psychiatric condition. One should note that memory impairment, although present in most people with dementia, is not an essential requirement; rather, at least two cognitive domains must be impaired, and of course the other criteria must be met. The presence of dementia without a dominant memory complaint has been demonstrated in large cohorts of patients with dementia $[14,15]$.

The 2011 NIA-AA revised core clinical criteria for dementia advance the clinical framework where dementia can be diagnosed when cognitive or behavioral symptoms meet the following [1]:

1. They interfere with the ability to function independently at work or at usual activities.

2 . They represent a decline from prior levels of functioning and performing.

3. They are not explained by delirium or major psychiatric disorder.

4. Cognitive impairment is detected and diagnosed through a combination of history-taking from the patient and a knowledgeable informant and of an objective cognitive assessment, either a bedside mental status examination or neuropsychological testing. Neuropsychological testing should be performed when the routine history and bedside mental status examination cannot provide a confident diagnosis.

5. The cognitive or behavioral impairment involves at least two of the following domains:

(a) Impaired ability to acquire and remember new information - symptoms include repetitive questions or conversations, misplacing personal belongings, forgetting events or appointments, and getting lost on a familiar route.

(b) Impaired reasoning and handling of complex tasks, poor judgment - symptoms include poor understanding of safety risks, inability to manage finances, poor decision-making ability, and inability to plan complex or sequential activities.

(c) Impaired visuo-spatial abilities - symptoms include inability to recognize faces or common objects or to find objects in direct view despite good acuity, and inability to operate simple implements or orient clothing to the body.

(d) Impaired language functions (speaking, reading, writing) - symptoms include difficulty thinking of common words while speaking, hesitations, and speech, spelling and writing errors.

(e) Changes in personality, behavior or comportment - symptoms include impaired motivation, initiative, increasing apathy, loss of drive, social withdrawal, decreased interest in previous activities, loss of empathy, compulsive or obsessive behaviors, and socially unacceptable behaviors. 
These NIA-AA clinical criteria were designed to be flexible enough to be used by both general healthcare providers without access to neuropsychological testing, advanced imaging and CSF measures as well as researchers and those at specialty clinics

\section{Proposals adopted at the Fourth CCCD}

We recommend the adoption of the criteria for dementia proposed by the 2011 NIA-AA working group.

\section{Alzheimer's disease dementia}

The clinical diagnosis of AD dementia has traditionally rested on a two-step process: identifying the presence of dementia and then a clinical pattern of AD conforming to the 1984 National Institute of Neurological and Communicative Disease and the Alzheimer's Disease and Related Diseases Association criteria [16]. Indeed, the National Institute of Neurological and Communicative Disease criteria have been the diagnostic standard for more than 25 years, with many validation studies against neuropathological gold standards having been performed [17]. The current NIA-AA criteria propose some revisions to the National Institute of Neurological and Communicative Disease framework. In addition to the clinically determined probable and possible AD diagnosis that have been used until now, a third grouping of 'probable or possible $\mathrm{AD}$ with biomarker evidence of $\mathrm{AD}-\mathrm{P}$ ' has been added [1]. The use of biomarkers to enhance certainty of $\mathrm{AD}$ is described presently as useful in three circumstances: investigational studies, clinical trials, and optionally where the biomarkers are available and deemed appropriate by the clinician. We will discuss this important third category at length below and in the other papers of the consensus conference.

Within the revised probable AD dementia grouping there is recognition that there are both amnestic and nonamnestic presentations that can be clinically identified. The amnestic presentation, which is most common, has both impairment in memory function as well as in a second cognitive domain, while the less common nonamnestic presentations can include progressive visuospatial impairment, syndromes of predominant frontal/ executive dysfunction, or language impairments with prominent naming difficulties. Individuals diagnosed as probable $\mathrm{AD}$ by the previous criteria remain probable AD by the current revised criteria.

The diagnostic designation of possible AD continues to include individuals with atypical course or clinical features including the presence of concomitant cerebrovascular disease, manifestations of dementia with Lewy bodies (including visual hallucinations, fluctuations or parkinsonian features) or the presence of another neurological or psychiatric disease comorbidity, or medication use that could be contributing to the dementia.
The third category of 'probable or possible AD with biomarker evidence' includes either evidence of brain amyloidosis with low CSF A $\beta 42$ or positive PET amyloid imaging or, alternatively, downstream evidence of neuronal degeneration or injury with evidence of elevated CSF tau (total tau and/or phosphorylated tau); decreased FDG uptake on PET in the temporo-parietal cortex; or disproportionate atrophy on structural MRI in the medial, basal and lateral temporal lobe, and medial parietal cortex. This biomarker evidence is proposed to increase the certainty that the basis of the clinical dementia syndrome is the AD-P. Given that these biomarkers have not yet been approved for diagnostic use by most regulatory or paying authorities in Canada, their use will be restricted in the near term to research studies. At the same time, it must be acknowledged that in a few centers and provinces (for example, Quebec) the biomarkers are already available in certain circumstances for specialist physicians.

\section{Proposals adopted at the Fourth CCCD}

We recommend the adoption of the recommendations concerning probable and possible $\mathrm{AD}$ dementia core clinical diagnostic criteria proposed by the 2011 NIA-AA working group.

\section{Mild cognitive impairment and prodromal Alzheimer's disease}

There is overwhelming evidence that MCI is a risk state for progression to $\mathrm{AD}$ dementia. The development of AD-P biomarkers has supported a more refined clinicobiological definition of this at-risk state. Both the NIAAA working group and the IWG have undertaken to integrate biomarkers into the diagnostic formulation of this condition. The NIA-AA group has conceptualized their approach around $\mathrm{MCI}$ due to $\mathrm{AD}$, with the presence of AD-P biomarkers increasing the probability of progression to $\mathrm{AD}$ dementia. The IWG has taken a different view that a characteristic clinical presentation of $A D$ in the predementia stage (prodromal AD), supported by the presence of one or more AD-P biomarkers, is sufficient to diagnose $\mathrm{AD}$. While there are currently no proven therapies to influence the longer term outcomes of these conditions, both definitions support the research of predementia $\mathrm{AD}$ interventions, both pharmacological and nonpharmacological. We will review each proposal in detail below.

\section{The International Working Group definition of prodromal Alzheimer's disease}

The IWG has taken the view that 'the term "Alzheimer's Disease" should ... encompass the whole spectrum of its clinical course, from earliest specific clinical symptoms, to dementia' ([5], p. 1120). Importantly, this allows a 
diagnosis of AD independent of a diagnosis of dementia. Prodromal AD would be the predementia stage of $\mathrm{AD}$, in which there is a typical AD-type clinical amnestic phenotype, along with positive AD-P biomarker(s). The clinical amnestic phenotype includes the presence of definite impairment on specified memory tests that control for encoding and where there is a failure to normalize recall with cuing (for example, impaired performance on the Free and Cued Selective Reminding Test). There needs to be supporting evidence of at least one abnormal AD-P biomarker for a patient to be reclassified from MCI (a risk state) to prodromal $\mathrm{AD}$ (a stage of $\mathrm{AD}$ ). Alternatively, atypical $\mathrm{AD}$ can be diagnosed in its prodromal stages when a known phenotype of AD (posterior cortical degeneration, logogenic aphasia, or frontal behavioral variant) is supported by one or more abnormal AD-P biomarkers. AD dementia transition occurs when cognitive symptoms become sufficiently severe to interfere with social functioning and instrumental activities of daily living $[4,5]$. The prodromal phase of the disease describes the predementia stage and AD dementia describes the later stages.

The NIA-AA definition of mild cognitive impairment due to Alzheimer's disease

The NIA-AA group has taken a different approach, applying a four-step process to establish the framework for a diagnosis of MCI due to AD [2]. They characterize the first step as establishing the presence of MCI, building on the published literature of the past 15 years. The second step involves the characterization of the cognitive and functional measures for assessment to establish the diagnosis. The third step considers establishing an etiological diagnosis of MCI of the $\mathrm{AD}$ type based on ruling out vascular, traumatic, and other medical causes of MCI, looking for evidence of progressive decline, and looking at $\mathrm{AD}$ genetic factors where available. The final step addresses the presence of positive biomarkers for $\mathrm{AD}-\mathrm{P}$, classifying these as biomarkers of brain $\mathrm{A} \beta$ protein deposition or biomarkers of downstream neuronal degeneration or injury.

In the first step, there were subtle but important specifications for the clinical core criteria for a diagnosis of MCI. There should be concern from either the individual or their informant regarding a change in cognition, compared with their previous level of functioning, with objective evidence of lower than expected performance in one or more cognitive domains. The cognitive changes would typically be 1 to 1.5 standard deviations below appropriate age and education-adjusted normative data. These ranges are guidelines and not cutoff scores. On the important issue of functional disability, within the proposed criteria there is a description of 'generally mild functional impairment for complex tasks, but basic ADLs [activities of daily living] should be preserved' ([2], p. 4). Individuals should not be diagnosed with dementia in that 'there is no evidence of a significant impairment in social or occupational functioning' ([2], p. 3) and that there should be preservation of independence in functional abilities. This important acknowledgement that persons with MCI commonly have mild problems performing complex functional tasks they were able to perform previously represents a further elaboration of a difficult area in the diagnosis: 'They may take more time, be less efficient, and make more errors at performing such activities than in the past. Nevertheless, they generally maintain their independence of function in daily life, with minimal aids or assistance' ([2], p. 3). The critical factor separating MCI and dementia (presumably AD) in the NIA-AA framework consists of the severity and extent (one versus at least two domains) of the cognitive/behavioral deficit, and consists of the presence or not of a significant degree of functional impairment. Unfortunately, despite these efforts to define the nature of the functional disability, it remains a difficult issue, depending on how performance of daily activities is assessed, the quality of the caregiver/ families' responses, and the nature of the activities that exist to interrogate.

The final step in the NIA-AA approach to MCI is the application of biomarkers within research criteria of $\mathrm{MCI}$ due to $\mathrm{AD}$. A nomenclature is proposed where the use of biomarkers can increase the likelihood of MCI due to AD in any individual. If both $A \beta$ and neuronal injury (tau, FDG, or structural MRI) biomarkers are positive - that is, indicating AD-P - then a high likelihood that MCI syndrome is due to AD therefore exists. If one of the two categories of biomarker is untested and the other is positive, then the likelihood is intermediate. In cases where the two results are conflicting (that is, $A \beta$ markers suggest AD-P, but neuronal injury biomarkers do not), the results are ambiguous, or the tests have not been carried out, then the biomarkers are deemed uninformative and only MCI core clinical criteria would be met.

An important caveat has been provided by the NIA-AA group around the intended application of the MCI due to AD criteria. The core clinical criteria for MCI are designed to be applicable in all clinical settings, but the research criteria with biomarkers are currently restricted to research because of lack of standardization of biomarkers, limited experience with cutoff points for diagnosis, and limitations in access. The research criteria are designed to be 'a work-in-progress that will be updated regularly as new information becomes available' ([2], p. 2).

The NIA-AA approach to MCI appears more conservative than that of the IWG, making fewer assumptions. 
We note again that IWG criteria are intended as research criteria where the NIA-AA criteria include clinical application, and are compatible with care settings where there is no access to biomarkers. When biomarker tests are not available, the core clinical criteria for MCI are deemed sufficient. Indeed, there is no suggestion that more than this should be done clinically at the present time. Even were the new MCI due to AD criteria to become used in clinical settings, the only change would be adding levels of likelihood of AD etiology in view of added biomarker information. In fact, for both $\mathrm{AD}$ and $\mathrm{MCI}$, the core clinical criteria are separated out and given primacy. One can continue to do adequate medicine without recourse to FDG PET, amyloid imaging, structural MRI, or CSF testing.

The concept of prodromal AD in the Dubois criteria, in contrast, requires the application and cost of ancillary biomarkers. Were these criteria to be applied in clinical settings, patients might be requesting these tests prematurely given current caveats around their use. The advantage of being reclassified from MCI to a more accurate designation as prodromal AD is currently uncertain. Nevertheless, such a shift could lead to increasing referrals to specialty clinics in order to gain access to cutting edge sophisticated neuroimaging as well as CSF biomarkers. MCI remains the more clinically accessible diagnostic clinical construct at the present time.

Functional impairment remains a key dividing line between predementia and dementia states. Unfortunately, assessing functional decline is difficult, depends largely on how carefully performance of daily activities is assessed, the quality of the caregiver/families' responses, and what activities there actually exist to interrogate. Some prominent researchers such as David Bennet have suggested abandoning function questions completely and developing algorithms based on cutoff scores on normed cognitive measures to designate $\mathrm{AD}$, and this appears actually to correlate as accurately with postmortem pathology [18]!

If function is preserved completely, then the individual clearly shows MCI. But how much functional impairment is necessary to be significantly impaired? Recently, Morris has argued that with the new criteria there was somehow more leeway for functionally impaired individuals to be labeled with MCI rather than dementia [19]. Morris complains that if a liberal interpretation is applied, individuals who are only mildly impaired on a range of functions might still be considered to be functionally independent, and therefore are classified with MCI. He reviewed data suggesting that indeed most mild AD subjects could be reclassified as MCI in this manner. We reject this position as overly alarmist, and consider the current suggestions to be appropriate and feasible. The authors of the NIA-AA papers stress that 'the clinical syndrome (of MCI) is almost identical to the one previously described by Petersen et al' ([2], p. 4). While functional impairment will continue to be a difficult and vague criterion, we doubt that the new description will change in the least the clinical approach to $\mathrm{MCI}$ and dementia diagnosis.

\section{Proposals adopted at the Fourth CCCD}

We recommend the adoption of the core clinical criteria for MCI proposed by the 2011 NIA-AA working group [2].

We recommend reassessment of the utility of the concept of prodromal AD in the future when AD-P biomarkers are available, validated, and approved for use in Canada.

\section{Preclinical stages of Alzheimer's disease and normal older subjects}

An important contribution of both research working groups was to open the discussion on the lexicon and approach to research with cognitively normal subjects who have abnormal AD-P biomarkers. These subjects would be hypothesized as being in the long preclinical phase of $\mathrm{AD}$ preceding $\mathrm{MCI}$, where there is evolving AD-P, particularly $A \beta$ peptide accumulation and initial neuronal injury. The NIA-AA working group on preclinical AD addressed this thorny but important issue, with the proposal of a draft operational research framework for detecting and staging preclinical AD [3]. They liken their proposal to preclinical disease conditions in other medical specialties - for example, carcinoma in situ or type II diabetes - where it is accepted that symptoms are not necessary to diagnose disease. The operational framework is based on the growing ability to detect abnormal biomarkers in individuals who might otherwise be classified as older normals. Considerable evidence indicates that there are between 20 and $40 \%$ of clinically normal older individuals who demonstrate evidence of amyloidopathy [20] and there is reason to think that positive amyloid biomarkers on imaging precede dementia by 10 to 15 years [21]. There is also a set of older individuals who show evidence of neuronal degeneration, with changes on MRI or FDG PET. There are even individuals who show both of these, along with very subtle cognitive changes but no real complaints of memory loss. These individuals do not meet the criteria for MCI.

The NIA-AA group proposes an operational research framework for staging preclinical AD. Stage 1 preclinical $\mathrm{AD}$ is defined as asymptomatic amyloidosis; Stage 2 is defined as asymptomatic amyloidosis accompanied by evidence of neurodegeneration (evidence of elevated CSF tau, decreased FDG uptake on PET in the temporoparietal cortex; or disproportionate atrophy on structural 
MRI); and Stage 3 is defined with the same biomarker, with colleagues underscoring that the framework is for research and is not intended for clinical diagnostic purposes, and that many individuals meeting criteria for the three stages may never actually develop the clinical features of $\mathrm{AD}$ in their lifetime. They acknowledge that 'the definitive studies to determine whether the majority of asymptomatic individuals with evidence of AD-P are indeed destined to develop $\mathrm{AD}$ dementia ... will likely take more than a decade to fully accomplish' ([3], p. 11).

The IWG applies the more cautious definition of asymptomatic at-risk for $\mathrm{AD}$ to cognitively normal individuals with positive pathophysiological markers including brain amyloidosis, and reserves presymptomatic $\mathrm{AD}$ for individuals identified as affected by autosomal dominant $\mathrm{AD}$ mutations and therefore certain to develop AD symptoms in the future [5]. This approach acknowledges explicitly that AD-P in the absence of clinical symptoms should not be seen as a disease state, and should not be diagnosed as such. Consistent with the philosophy of AD as a clinical-biological entity, the IWG would consider shifting the border between normal and disease only if there were validated very early cognitive or behavioral changes that predate the features of prodromal $\mathrm{AD}$, and that co-occur with AD-P abnormalities.

\section{Proposals adopted at the Fourth CCCD}

We recommend the IWG definition of asymptomatic atrisk for AD states [5] for research purposes.

Given that the presence of brain amyloid in normal people is of uncertain significance, we propose that the CCCD discourages the use of amyloid deposition imaging in individuals without memory loss, outside the research setting. The medical community should be clear in its discussions with patients, the media and the general population that a presence of brain amyloid in normal people is of unclear significance at the present time.

\section{Vascular dementia and vascular cognitive impairment}

Vascular dementia occurring with $\mathrm{AD}$ is common, but pure vascular dementia appears to be uncommon [22,23]. A full review of the management of dementia with a cerebrovascular component was developed in Canada in the Third CCCD and was presented by Bocti, Black, and Frank [24]. There are both conceptual and pragmatic difficulties in trying to portray vascular dementia as a distinct entity. Of the criteria now in use, the National Institute of Neurological Disorders and Stroke-Association Internationale pour la Recherché et l'Enseignement en Neurosciences criteria [22] and the Alzheimer's Disease Diagnostic and Treatment Centers criteria [25] have been especially influential but each set has limitations. In practice, most criteria are insensitive [26] and different sets of criteria give differing estimates of who has vascular dementia [26,27]. Vascular lesions are found in many dementia patients, including those with otherwise classical $\mathrm{AD}$, where they are often detected only by routine neuroimaging. On the contrary, patients with only vascular pathology as the cause of their dementia have been uncommon in many series $[28,29]$. Most patients have mixed pathology, so that patients with vascular lesions commonly have evidence of other neurodegenerative disorders [30]. One should also note that many patients with positive imaging do not have a stroke history, implying a high occurrence of silent strokes.

Against this background, the proposal to consider moving the concept from vascular dementia to one of VCI appears justified [31,32]. This led the AHA/ASA to form a working group that has issued a consensus position paper entitled 'Vascular Contributions to Cognitive Impairment and Dementia' in 2011 [8]. This consensus position paper proposes diagnostic criteria for VCI as well as several recommendations regarding neuroimaging, nonpharmacological and pharmacological management at different stages of the clinical spectrum. According to the AHA/ASA paper, VCI should include all stages of cognitive disorders associated with cerebrovascular disease, from mild symptoms to overt dementia: 'Simply put, VCI is a syndrome with evidence of clinical stroke or subclinical vascular brain injury and cognitive impairment affecting at least 1 cognitive domain' ([8], p. 4). The practical diagnostic approach presented has the merit of being independent from the specific underlying cerebrovascular process (cardioembolic, atherosclerotic, ischemic, and so forth). Diagnostic criteria are proposed for vascular dementia (probable and possible) and for vascular MCI (probable, possible, and unstable).

Based on cognitive testing of a minimum of four cognitive domains (executive function, memory, language, and visuo-spatial functions), the diagnosis of dementia requires a decline on at least two domains, sufficient to interfere with functioning. Probable vascular dementia requires the presence of a clear temporal relationship between a cerebrovascular event and cognitive impairment or a clear relationship between the severity and pattern of cognitive impairment and neuroimaging evidence of subcortical cerebrovascular disease. The diagnostic criteria for possible vascular dementia are fulfilled if there is no clear relationship between cognitive impairment and cerebrovascular disease, if the information available is insufficient or clinical symptoms preclude assessment, or if there is evidence of a concomitant neurodegenerative process that may contribute to the cognitive impairment. These criteria do not require the traditional stepwise progression of cognitive impairment associated with multi-infarct dementia, and they are not 
conditional on memory impairment being present. The diagnostic criteria for probable and possible vascular MCI parallel those of probable and possible vascular dementia, with the important exception that instrumental activities of daily living should be normal or only mildly impaired. Interestingly, the ASA/AHA introduced the concept of unstable vascular MCI to account for individuals whose impairment may revert to normal during follow-up.

\section{Proposals adopted at the Fourth CCCD}

We recommend the 2011 ASA/AHA recommendations for the diagnosis of VCI [8].

\section{What is missing? What is needed in the future?}

This paper has focused on the new diagnostic formulations of $\mathrm{AD}$ that support the earlier identification of disease based on biomarker integration. There is currently an emerging belief in the field that the failure to make progress in therapeutics of the disease is arising from interventions that occur too late in the processed pathophysiological process. The emergence of biomarkers that identify the pathology of AD ahead of its symptomatic expression may in future allow the potential for studies directed at earlier intervention, including those directed at prevention of $\mathrm{AD} /$ dementia.

The time may now be here to reconceptualize the functional impairment criteria for $\mathrm{AD}$ diagnosis given some of the significant decade-long challenges of defining the right amount of impairment. Defining functional impairment cutoff points is very difficult, and even assessing function in many cases is unworkable, given its significant social and gender context. This is clearly an area that requires further work.

Outside AD are the considerations of the status of mixed dementia versus pure AD and vascular dementia. In the clinic, the majority of older patients have some evidence of vascular as well as neurofibrillary pathology, and if we had (or will have in the future) better imaging techniques this fraction would probably rise. The McKhann group paper proposes designating etiologically mixed presentations of $\mathrm{AD}$ as a form of possible $\mathrm{AD}$ dementia, and the IWG refers to mixed AD for such individuals $[4,5]$. However, clear emphasis remains on pure $\mathrm{AD}$ as the major diagnosis because its pathological footprint remains most accessible. Other researchers consider that this approach should be turned on its head and mixed pathology considered the usual state [33], particularly in older individuals [26,34]. As Richards and Brayne state in a recent British Medical Journal editorial: 'It is timely to interrogate the term Alzheimer's Disease. In older age groups, $\mathrm{AD}$ seems to be a diffuse clinical syndrome representing the gradual accumulation of multiple pathologies, arising from multiple interlocking risk factors over the life course. The term Alzheimer's syndrome seems more appropriate' ([33], p. 865). This alternative approach bears empirical consideration and assessment in the future.

\section{Abbreviations \\ $A \beta$, amyloid beta; $A D$, Alzheimer disease; $A D-P$, Alzheimer disease pathophysiological process; AHA, American Heart Association; ASA, American Stroke Association; CCCD, Canadian Consensus Conference on the Diagnosis and Treatment of Dementia; CSF, cerebrospinal fluid; FDG, fludeoxyglucose; IWG, International Working Group; MCl, mild cognitive impairment; MRI, magnetic resonance imaging; NIA-AA, National Institute of Aging/Alzheimer's Association; PET, positron emission tomography; VCl, vascular cognitive impairment.}

Competing interests

The authors declare that they have no competing interests.

Acknowledgements

$\mathrm{HC}$ is supported by the Canadian Institutes of Health Research.

\section{Declarations}

This article has been published as part of Alzheimer's Research \& Therapy Volume 5 Supplement 1, 2013: Background documents to the 4th Canadian Consensus Conference on the Diagnosis and Treatment of Dementia (CCCDTD4). The full contents of the supplement are available online at http://alzres.com/supplements/5/S1.

Publication charges for the supplement were funded by the Canadian Consensus Conference on the Diagnosis and Treatment of Dementia (CCCDTD). Although residual conference funds used include contributions from pharmaceutical companies, no commercial organization has been involved in the selection of participants, choice of topics, preparation of background papers or recommendations. In kind support was also provided by the Canadian Dementia Knowledge Translation Network, and the offices of Drs Serge Gauthier (McGill University), Christopher Patterson (McMaster University) and Howard Chertkow (McGill University), whose role as Guest Editors involved the coordination of the project without involvement in the journal's standard peer review process which applied for all articles.

\section{Author details}

'Department of Neurology and Neurosurgery, McGill University, Montreal, Canada. ${ }^{2}$ Bloomfield Centre for Research in Aging, Lady Davis Institute, 3755 Cote St Catherine Road, Montreal, Canada H3T 1E2. ${ }^{3}$ Division of Neurology, Faculty of Medicine, University of British Columbia, UBC Hospital, S192-2211 Wesbrook Mall, Vancouver, Canada V6T 2B5. ${ }^{4}$ Division of Neurology, Faculty of Medicine, University of British Columbia, UBC Hospital, 2211 Wesbrook Mall, Rm 152, Vancouver, Canada V6T 2B5. ${ }^{5}$ Department of Medicine, Centre Hospitalier de I'Université de Montréal, 1560 rue Sherbrooke Est, Montreal, Canada H2L $4 \mathrm{M} 1$

Published: 8 July 2013

\section{References}

1. MCKhann GM, Knopman DS, Chertkow H, Hyman BT, Jack CR Jr, Kawas CH, Klunk WE, Koroshetz WJ, Manly JJ, Mayeux R, Mohs RC, Morris JC, Rossor MN, Scheltens P, Carrillo MC, Thies B, Weintraub S, Phelps CH: The diagnosis of dementia due to Alzheimer's disease: recommendations from the National Institute on Aging and the Alzheimer's Association workgroup. Alzheimers Dement 2011, 7:263-269.

2. Albert MS, Dekosky ST, Dickson D, Dubois B, Feldman HH, Fox NC, Gamst A, Holtzman DM, Jagust WJ, Petersen RC, Snyder PJ, Carrillo MC, Thies B, Phelps $\mathrm{CH}$ : The diagnosis of mild cognitive impairment due to Alzheimer's disease: recommendations from the National Institute on Aging and Alzheimer's Association workgroup. Alzheimers Dement 2011, 7:270-279.

3. Sperling RA, Aisen PS, Beckett LA, Bennett DA, Craft S, Fagan AM, Iwatsubo T, Jack CR Jr, Kaye J, Montine TJ, Park DC, Reiman EM, Rowe CC, Siemers E, Stern Y, Yaffe K, Carrillo MC, Thies B, Morrison-Bogorad M, Wagster MV, Phelps CH: Toward defining the preclinical stages of Alzheimer's disease: recommendations from the National Institute on Aging and the 
Alzheimer's Association workgroup. Alzheimers Dement 2011, 7:280-292.

4. Dubois B, Feldman HH, Jacova C, Dekosky ST, Barberger-Gateau P, Cummings J, Delacourte A, Galasko D, Gauthier S, Jicha G, Meguro K, O'Brien J, Pasquier F, Robert P, Rossor M, Salloway S, Stern Y, Visser PJ, Scheltens P: Research criteria for the diagnosis of Alzheimer's disease: revising the NINCDS-ADRDA criteria. Lancet Neurol 2007, 6:734-746.

5. Dubois B, Feldman HH, Jacova C, Cummings JL, Dekosky ST, BarbergerGateau P, Delacourte A, Frisoni G, Fox NC, Galasko D, Gauthier S, Hampel H, Jicha GA, Meguro K, O'Brien J, Pasquier F, Robert P, Rossor M, Salloway S, Sarazin M, de Souza LC, Stern Y, Visser PJ, Scheltens P: Revising the definition of Alzheimer's disease: a new lexicon. Lancet Neurol 2010, 9:1118-1127.

6. Jack CR, Jr, Lowe VJ, Weigand SD, Wiste HJ, Senjem ML, Knopman DS, Shiung MM, Gunter JL, Boeve BF, Kemp BJ, Weiner M, Petersen RC; Alzheimer's Disease Neuroimaging Initiative: Serial PIB and MRI in normal, mild cognitive impairment and Alzheimer's disease: implications for sequence of pathological events in Alzheimer's disease. Brain 2009, 132:1355-1365.

7. Jack CR, Jr, Knopman DS, Jagust WJ, Shaw LM, Aisen PS, Weiner MW, Petersen RC, Trojanowski JQ: Hypothetical model of dynamic biomarkers of the Alzheimer's pathological cascade. Lancet Neurol 2010, 9:119-128.

8. Gorelick PB, Scuteri A, Black SE, Decarli C, Greenberg SM, ladecola C, Launer LJ, Laurent S, Lopez OL, Nyenhuis D, Petersen RC, Schneider JA, Tzourio C, Arnett DK, Bennett DA, Chui HC, Higashida RT, Lindquist R, Nilsson PM, Roman GC, Sellke FW, Seshadri S; American Heart Association Stroke Council; Council on Epidemiology and Prevention; Council on Cardiovascular Nursing; Council on Cardiovascular Radiology and Intervention; Council on Cardiovascular Surgery and Anesthesia: Vascular contributions to cognitive impairment and dementia: a statement for healthcare professionals from the American Heart Association/American Stroke Association. Stroke 2011, 42:2672-2713

9. Morris JC: The Clinical Dementia Rating (CDR): current version and scoring rules. Neurology 1993, 43:2412-2414.

10. Nasreddine ZS, Phillips NA, Bédirian V, Charbonneau S, Whitehead V, Collin I, Cummings JL, Chertkow H: The Montreal Cognitive Assessment, MoCA: a brief screening tool for mild cognitive impairment. J Am Geriatr Soc 2005, 53:695-699.

11. American Psychological Association: Diagnostic and Statistical Manual of Mental Disorders, 4th ed.: DSM-IV. 4th edition. Washington, DC: American Psychological Association; 1994.

12. American Psychological Association: Text Revision of the Diagnostic and Statistical Manual of Mental Disorders, 4th ed.: DSM-IV-TR. Washington, DC: American Psychological Association; 2000.

13. Winblad B, Palmer K, Kivipelto M, Jelic V, Fratiglioni L, Wahlund LO, Nordberg A, Bäckman L, Albert M, Almkvist O, Arai H, Basun H, Blennow K, de Leon M, DeCarli C, Erkinjuntti T, Giacobini E, Graff C, Hardy J, Jack C, Jorm A, Ritchie K, van Duijn C, Visser P, Petersen RC: Mild cognitive impairment - beyond controversies, towards a consensus: report of the International Working Group on Mild Cognitive Impairment. J Intern Med 2004, 256:240-246.

14. Lopez OL, Becker JT, Klunk W, Saxton J, Hamilton RL, Kaufer DI, Sweet RA, Cidis Meltzer C, Wisniewski S, Kamboh MI, DeKosky ST: Research evaluation and diagnosis of probable Alzheimer's disease over the last two decades: I. Neurology 2000, 55:1854-1862.

15. Feldman H, Levy AR, Hsiung GY, Peters KR, Donald A, Black SE, Bouchard RW, Gauthier SG, Guzman DA, Hogan DB, Kertesz A, Rockwood K; ACCORD Study Group: A Canadian cohort study of cognitive impairment and related dementias (ACCORD): study methods and baseline results. Neuroepidemiology 2003, 22:265-274.

16. McKhann G, Drachman D, Folstein M, Katzman R, Price D, Stadlan EM: Clinical diagnosis of Alzheimer's disease: report of the NINCDS-ADRDA Work Group under the auspices of Department of Health and Human Services Task Force on Alzheimer's Disease. Neurology 1984, 34:939-944.

17. Mayeux R: Early Alzheimer's disease. N Engl J Med 2010, 362:2194-2201.

18. Bennett DA, Schneider JA, Aggarwal NT, Arvanitakis Z, Shah RC, Kelly JF, Fox $J \mathrm{H}$, Cochran EJ, Arends D, Treinkman AD, Wilson RS: Decision rules guiding the clinical diagnosis of Alzheimer's disease in two community-based cohort studies compared to standard practice in a clinic-based cohort study. Neuroepidemiology 2006, 27:169-176.
19. Morris JC: Revised criteria for mild cognitive impairment may compromise the diagnosis of Alzheimer disease dementia. Arch Neuro/ 2012, 69:700-708.

20. Morris JC, Storandt M, McKeel DW, Jr, Rubin EH, Price JL, Grant EA, Berg L: Cerebral amyloid deposition and diffuse plaques in 'normal' aging: evidence for presymptomatic and very mild Alzheimer's disease. Neurology 1996, 46:707-719.

21. Mintun MA, Larossa GN, Sheline YI, Dence CS, Lee SY, Mach RH, KlunkWE, Mathis CA, DeKosky ST, Morris JC: [ $\left.{ }^{11} \mathrm{C}\right] \mathrm{PIB}$ in a nondemented population: potential antecedent marker of Alzheimer disease. Neurology 2006, 67:446-452.

22. Roman GC, Tatemichi TK, Erkinjuntti T, Cummings UL, Masdeu JC, Garcia JH, Amaducci L, Orgogozo JM, Brun A, Hofman A, et al:: Vascular dementia: diagnostic criteria for research studies. Report of the NINDS-AIREN International Workshop. Neurology 1993, 43:250-260.

23. O'Brien JT, Erkinjuntti T, Reisberg B, Roman G, Sawada T, Pantoni L, Bowler JV, Ballard C, DeCarli C, Gorelick PB, Rockwood K, Burns A, Gauthier S, DeKosky ST: Vascular cognitive impairment. Lancet Neurol 2003, 2:89-98.

24. Bocti C, Black SE, Frank C: Management of dementia with a cerebrovascular component. Alzheimer Dement 2007, 3:398-403.

25. Chui HC, Victoroff Jl, Margolin D, Jagust W, Shankle R, Katzman R: Criteria fo the diagnosis of ischemic vascular dementia proposed by the State of California Alzheimer's Disease Diagnostic and Treatment Centers. Neurology 1992, 42(3 Pt 1):473-480.

26. Rockwood K, Davis H, MacKnight C, Vandorpe R, Gauthier S, Guzman A, Montgomery P, Black S, Hogan DB, Kertesz A, Bouchard R, Feldman H: The Consortium to Investigate Vascular Impairment of Cognition: methods and first findings. Can J Neurol Sci 2003, 30:237-243.

27. Gold G, Bouras C, Canuto A, Bergallo MF, Herrmann FR, Hof PR, Mayor PA, Michel JP, Giannakopoulos P: Clinicopathological validation study of four sets of clinical criteria for vascular dementia. Am J Psychiatry 2002, 159:82-87.

28. Holmes C, Cairns N, Lantos P, Mann A: Validity of current clinical criteria for Alzheimer's disease, vascular dementia and dementia with Lewy bodies. Br J Psychiatry 1999, 174:45-50.

29. Knopman DS, DeKosky ST, Cummings JL, Chui H, Corey-Bloom J, Relkin N, Small GW, Miller B, Stevens JC: Practice parameter: diagnosis of dementia (an evidence-based review). Report of the Quality Standards Subcommittee of the American Academy of Neurology. Neurology 2001 56:1143-1153.

30. Tomlinson BE, Blessed $\mathrm{G}$, Roth M: Observations on the brains of demented old people. J Neurol Sci 1970, 11:205-242.

31. Hachinski V: Preventable senility: a call for action against the vascular dementias. Lancet 1992, 340:645-648.

32. Pike KE, Savage G, Villemagne VL, Ng S, Moss SA, Maruff P, Mathis CA, Klunk WE, Masters CL, Rowe CC: Beta-amyloid imaging and memory in nondemented individuals: evidence for preclinical Alzheimer's disease. Brain 2007, 130:2837-2844.

33. Richards M, Deary IJ: A life course approach to cognitive reserve: a model for cognitive aging and development? Ann Neurol 2005, 58:617-622.

34. Brayne C, Richardson K, Matthews FE, Fleming J, Hunter S, Xuereb JH, Paykel E, Mukaetova-Ladinska EB, Huppert FA, O'Sullivan A, Dening T; Cambridge City Over-75s Cohort Cc75c Study Neuropathology Collaboration: Neuropathological correlates of dementia in over-80-year-old brain donors from the population-based Cambridge City over-75s Cohort (CC75C) Study. J Alzheimers Dis 2009, 18:645-658.

35. Richards M, Brayne C: What do we mean by Alzheimer's disease? BMJ 2010, 341:c4670.

\section{doi:10.1186/alzrt198}

Cite this article as: Chertkow $\mathrm{H}$, et al: Definitions of dementia and predementia states in Alzheimer's disease and vascular cognitive impairment: consensus from the Canadian conference on diagnosis of dementia. Alzheimer's Research \& Therapy 2013, 5(Suppl 1):S2. 